\title{
PIM/FLT3 Kinase Inhibitor SEL24
}

National Cancer Institute

\section{Source}

National Cancer Institute. PIMIFLT3 Kinase Inhibitor SEL24. NCI Thesaurus. Code C133540.

An orally available inhibitor of PIM family serine/threonine protein kinases and mutant forms of FMS-related tyrosine kinase 3 (FLT 3; ST K1) with potential antineoplastic activity. PIM/FLT 3 kinase inhibitor SEL24 binds to and inhibits the kinase activities of PIM-1, -2 and -3 , and mutant forms of FLT3, which may result in the interruption of the G1/S phase cell cycle transition, an inhibition of cell proliferation, and an induction of apoptosis in tumor cells that overexpress PIMs or express mutant forms of FLT3. FLT3, a tyrosine kinase receptor that is overexpressed or mutated in various cancers, plays a role in signaling pathways that regulate hematopoietic progenitor cell proliferation, and in leukemic cell proliferation and survival. PIM kinases, downstream effectors of many cytokine and growth factor signaling pathways, including the FLT3 signaling pathway, play key roles in cell cycle progression and apoptosis inhibition and may be overexpressed in various malignancies. 